\title{
Turbulence assails fifth force
}

\section{Claims that gravitational measurements in the 1920 s can embody evidence for novel short-range forces could have a more humdrum explanation.}

THE saga of the 'fifth force' continues, but in an uncertain direction. The story, less than a year old, goes back to the reanalysis by Fischbach et al. (Phys. Rev. Lett. 53, 3; 1986) of the measurements by Eötvõs and associates in the 1920s of the gravitational attraction of several different kinds of material by the Earth. Although the old data have been taken for the past half century as a proof that gravity depends only on mass and not, for example, on chemical composition, they have errors (of the order of one part in $10,000)$ which appear to vary systematically with chemical composition. Is that correlation real, or just an artefact?

The little bombshell devised by Fischbach et al. began with the observation that the systematic errors in the old data appear to vary with the baryon content, in this context the nucleon content, of the materials used by Eötvons. The point is that the baryon content, or in this context simply the nucleon content, varies with atomic number; the greater the atomic number, the greater the number of nucleons per unit mass of material, largely on account of the nuclear packing fraction but also because the greater ratio of neutrons to protons in heavier nuclei means that the small electron mass makes a lesser contribution to the total.

But why should baryon number be what matters? In the contemporary fashion, Fischbach et al. interpreted the systematic variation of apparent gravitational attraction as the consequence of the exchange between baryons of low-mass, perhaps massless, particles; the shadowy creature known as the axion is one candidate. The outcome of the re-interpretation was that there would be a force resembling gravitation between all pairs of objects that would nevertheless fall off exponentially with distance (over a range measured in tens of metres) and that would be determined not by the product of the two masses but by the product of their baryon numbers, which is nearly, but not quite, the same thing (see Nature 319, 173; 1986).

Inevitably, the appearance of Fischbach et al. began a hearty buzz. Reactions have ranged from disbelief to solemn speculation about the implications of the new force, the fifth force (supplementing the two nuclear forces, electromagnetism and gravity), for theories of particle physics.

Among experimentalists, there has been a spate of proposals for making more sensitive measurements than were pos- sible in 1922. Some of the best proposals would not be directly concerned with variations of chemical composition, but are designed to separate the short-range fifth force (which is, by the way, repulsive) from long-range gravity by measurement of the variation of apparent gravitational forces with distance in the neighbourhood of known density inhomogeneities, as on the face of a cliff.

Suggestions that apparent gravity may not be exactly that specified by Newton's inverse-square law are by no means new. More than a decade ago, for example, Daniel R. Long described a series of measurements apparently showing that gravitational forces are an increasing function of distance over a range of tens of centimetres (Nature 260, 417; 1976), but several attempts to confirm this conclusion have not done so. More recently, F. D. Stacey from the University of Queensland has argued that measurements with a gravimeter in an Australian borehole provide evidence for departures from newtonian attraction at distances of the order of metres (see Stacey, F. D. \& Tuck, G. J., Nature 292, 230; 1981), although the promised repetition of that measurement in a tract of deep ocean (where density variations are probably better known) seems not yet to have materialized. At the beginning of this year, it may be thought, the time was ripe for radical innovation in the field.

Now the small and esoteric community of those concerned with the measurement of gravity will be in a tizzy all over again. S. Y. Chu from the University of California, Riverside, and R.H. Dicke from Princeton University have now put out the claim that the systematic errors in the original Eötvõs experiments are not the consequences of a fifth force but of a much more mundane phenomenon: thermal convection (Phys. Rev. Lett. 57, 1823; 1986). What they have seized on is the simple fact that the physical bulk of a sample suspended on an Eötvõs balance will depend on the atomic number of its constituents, as will the physical cross-section of the sample presented to thermally driven movements of the atmosphere in the neighbourhood of the equipment, simple draughts in the laboratory.

With only a little special pleading, Chu and Dicke are able to show that the conventional forces thus generated might account for the systematic errors. Fischbach et al. admit that there is a case to answer. It is interesting that Robert Dicke who has the reputation of an iconoclast, should on this occasion be defending the status quo.

Experimentalists whose grant applications are even now being judicially considered need not despair. Few will risk taking sides on the evidence available. The difference between the two groups demonstrates most clearly of all that the original data-set, however convincing it may have been in the 1920 s as a proof that the gravitational attraction between masses is independent of their chemical composition, is entirely inadequate for the purposes for which it is now being used, the attempt to understand the origins of the systematic errors.

The Eötvõs experiments involved a torsion balance designed so that dissimilar materials could be suspended from the two arms. There appear to have been three series of measurements, one in which the gravitational attraction of the Earth for three materials $(\mathrm{Cu}, \mathrm{Mg}-\mathrm{Al}$ alloy and "snakewood" (sic)) was compared with that for platinum, another in which an unreacted mixture of $\mathrm{AgSO}_{4}$ and $\mathrm{FeSO}_{4}$ was compared with itself after chemical reaction and a third series in which the attraction of pairs of materials are compared directly with each other.

In total, there are only nine pairs of measurements, two of which were omitted from the original analysis by Fischbach $e t$ al. while a third (involving radium) is omitted from the analysis by $\mathrm{Chu}$ and Dicke. Chu and Dicke have done well to extract from the data a correlation between the surface area of the original samples and the departures from the inverse-square law recorded in the 1920s. Fischbach et al. rightly say that there are features of the analysis by Chu and Dicke that cannot be unambiguously resolved at this late stage; the general opinion is that there will have to be more experiments.

To judge from what people say, there will be no shortage of them. Fischbach et al. have put gravity measurements on the map again. Such a development can only be worthwhile; it may even contribute to the easing of what remains a blot on the face of physics, that the gravitational constant ('big' $G$ ) remains uncertain to about one part in 5,000. John Maddox

A fuller account of this issue, by Alvaro de Rujula of CERN, will appear shortly in the News and Views pages. 